WellBeing International

WBI Studies Repository

2-2006

\title{
Animal Carcinogenicity Studies: 3. Alternatives to the Bioassay
}

Andrew Knight

Animal Consultants International

Jarrod Bailey

University of Newcastle-upon-Tyne

Jonathan Balcombe

Independent Scientist and Author

Follow this and additional works at: https://www.wellbeingintlstudiesrepository.org/acwp_arte

Part of the Animal Experimentation and Research Commons, Animal Studies Commons, and the Other Medical Sciences Commons

\section{Recommended Citation}

Knight, A., Bailey, J., \& Balcombe, J. (2006). Animal carcinogenicity studies: 3. Alternatives to the Bioassay. ATLA-NOTTINGHAM-, 34(1), 39.

This material is brought to you for free and open access by WellBeing International. It has been accepted for inclusion by an authorized administrator of the WBI Studies Repository. For more information, please contact wbisr-info@wellbeingintl.org.

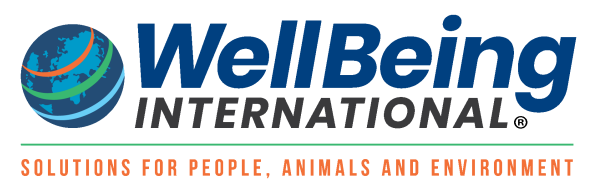




\title{
Animal Carcinogenicity Studies: 3. Alternatives to the Bioassay
}

\author{
Andrew Knight, ${ }^{1}$ Jarrod Bailey² and Jonathan Balcombe ${ }^{3}$ \\ ${ }^{1}$ Animal Consultants International, London, UK; ${ }^{2}$ School of Population and Health Sciences, Faculty of \\ Medical Sciences, University of Newcastle upon Tyne, Newcastle upon Tyne, UK; 3 Physicians Committee for \\ Responsible Medicine, Washington DC, USA
}

\begin{abstract}
Summary - Conventional animal carcinogenicity tests take around three years to design, conduct and interpret. Consequently, only a tiny fraction of the thousands of industrial chemicals currently in use have been tested for carcinogenicity. Despite the costs of hundreds of millions of dollars and millions of skilled personnel hours, as well as millions of animal lives, several investigations have revealed that animal carcinogenicity data lack human specificity (i.e. the ability to identify human non-carcinogens), which severely limits the human predictivity of the bioassay. This is due to the scientific inadequacies of many carcinogenicity bioassays, and numerous serious biological obstacles, which render profoundly difficult any attempts to accurately extrapolate animal data in order to predict carcinogenic hazards to humans. Proposed modifications to the conventional bioassays have included the elimination of mice as a second species, and the use of genetically-altered or neonatal mice, decreased study durations, initiation-promotion models, the greater incorporation of toxicokinetic and toxicodynamic assessments, structure-activity relationship (computerised) systems, in vitro assays, cDNA microarrays for detecting changes in gene expression, limited human clinical trials, and epidemiological research. The potential advantages of nonanimal assays when compared to bioassays include the superior human specificity of the results, substantially reduced time-frames, and greatly reduced demands on financial, personnel and animal resources. Inexplicably, however, the regulatory agencies have been frustratingly slow to adopt alternative protocols. In order to decrease the enormous cost of cancer to society, a substantial redirection of resources away from excessively slow and resource-intensive rodent bioassays, into the further development and implementation of non-animal assays, is both strongly justified and urgently required.
\end{abstract}

Key words: alternative, animal experiment, animal test, bioassay, cancer prevention, carcinogenicity, chemical classification, chemical safety, computer simulation, in vitro, risk assessment.

Address for correspondence: A. Knight, Animal Consultants International, 91 Vanbrugh Court, Wincott Street, London SE11 4NR, UK.

E-mail: info@animalconsultants.org

\section{Introduction}

Due to limited human exposure data, the identification and regulation of exposure to potential human carcinogens has relied heavily on animal carcinogenicity studies, of which several thousand have been conducted since the first chemical bioassay in 1915 (1). However, surveys of the US Environmental Protection Agency (EPA) and National Toxicology Program (NTP) toxic chemical databases and the International Agency for Research on Cancer (IARC) Monographs series, and the findings of other investigators, have all illustrated the poor human specificity (the ability to identify human non-carcinogens), and hence the poor human predictivity, of animal carcinogenicity data $(2-10)$.

Alternatives to the conventional rodent carcinogenicity bioassay exist, which offer superior human specificity and require substantially less time and expense. We present a review of the current status and likely future development of alternative assays as described in the peer-reviewed biomedical literature. We propose an alternative carcinogenicity testing protocol, highlight developments which deserve further research and investment, and offer suggestions designed to aid regulatory implementation.

\section{Methods}

We surveyed the peer-reviewed biomedical literature to locate papers describing bioassay alternatives which exist or are in the course of development. The "Medline" bibliographic biomedical database was searched by using the following search terms and combinations of them: alternative, animal, bioassay, carcinogenicity, chemical, computer, Environmental Protection Agency, experiment, International Agency for Research on Cancer, in vitro, mice, mouse, rat, rodent, simulation, test and regulation. Additional relevant papers were sourced from the reference lists of papers thus located. 


\section{Results and Discussion}

\section{Bioassay modifications}

Proposed modifications to the conventional rodent bioassay have included the elimination of mice as a second species, the use of genetically-altered or neonatal mice, decreased study durations, initiation-promotion models, and greater incorporation of toxicokinetic and toxicodynamic assessments.

\section{Eliminating the use of mice}

Mice have long been thought to be particularly poor indicators of potential human carcinogens (11). In their survey of the 254 chemicals within the comprehensive Berkeley-based Carcinogenic Potency Database (CPDB) that were positive in at least one rodent sex-species group, Gold et al. (6), found that 85-90\% would have been identified by choosing a single sex from each species. The most sensitive combination appeared to be male rats with either sex of mice. Also, after reviewing a wide range of studies, Alden et al. (12) were unable to locate an example of a mouse tumourigenic finding that predicted or confirmed a probable human response with negative findings in a rat bioassay. In surveying all the pharmaceuticals tested for carcinogenicity for which a marketing authorisation had been applied for in Germany and The Netherlands since 1980, Van Oosterhout et al. (13) found no instances of chemicals causing mouse-specific tumours that ever led to regulatory restriction or even precautionary labelling. Similarly, a negative mouse study was rarely used to declare positive rat findings irrelevant to humans. Consequently, these investigators endorsed earlier suggestions $(11,14)$ that mice should be eliminated from the standard two rodent species bioassay protocol.

\section{Genetically-altered mice}

Various transgenic and knockout mice have been described as potentially suitable for regulatory purposes (15-17). Transgenic mice have activated oncogenes (that facilitate neoplasia) introduced into their genomes, while knockout mice have tumour suppressor genes deleted. However, these models have not been validated, although at least one regulatory agency, the US Food and Drug Administration, is accepting transgenic mouse data as part of the safety assessment for selected pharmaceuticals (18). Cohen (19) assessed the use of two transgenic (TgAC, TgrasH2) and three knockout (heterozygous p53, homozygous XPA and homozygous XPA-heterozygous p53) genetically-modified mouse models by means of exposure to 21 chemicals, including geno- toxic and non-genotoxic chemicals, carcinogens and non-carcinogens. He concluded that, while these models might be suitable as screening assays, they did not offer definitive specificity for human carcinogens, nor could they definitively distinguish between genotoxic and non-genotoxic carcinogens. However, Goodman (15) asserted that genetically-altered mice generally exhibit the ability to detect genotoxic compounds, although, in most cases, these would also be detected by a standard battery of in vitro genotoxicity tests, rendering the in vivo tests unnecessary.

\section{Neonatal mice}

The short-term to medium-term neonatal mouse bioassay has been used experimentally in various forms since 1959 , to test a large number of chemicals. Neonatal mice have been shown to be very sensitive to genotoxic carcinogens, but not to carcinogens acting via epigenetic mechanisms (20).

\section{Shortening the bioassay}

Several investigators have criticised the two-year duration of the traditional rodent bioassay. Given that over $80 \%$ of rodent carcinogens are detectable within 12 months, Grice and Burek (21) were among the first to advocate a shortened 12-month protocol. Davies et al. (22) analysed 210 rodent carcinogens from Volumes 1-70 of the IARC Monographs series, and found that $66 \%$ of chemicals tested showed tumourigenic effects within the first 12 months of exposure, that $93 \%$ did so within the first 18 months, and that most of the tumour types not detected in the first 18 months were of "dubious relevance" to human risk assessment. Hence, they concluded that rodent bioassays should not exceed 18 months duration. Although longer study durations may afford an increased chance of detecting less-potent carcinogens, they are complicated by spontaneous tumours and other rodent geriatric pathology, such as hepatic or renal disease. Goodman (15) concluded: that carcinogenicity testing in rats, coupled with an assessment of toxicokinetic considerations (absorption, tissue distribution, metabolism and excretion) and an assessment of genotoxic potential, provides a sound carcinogenic evaluation; that mice are usually unnecessary; and that the duration of rodent bioassays should be shortened to 18 months.

\section{Initiation-promotion models}

Exposure to a carcinogen during tumour initiation can be followed by prolonged exposure to a non-carcinogenic promoter, or vice-versa, thereby hastening the process of carcinogenesis and potentially 
shortening the bioassay (23). In 1997, the International Conference on the Harmonisation of Technical Requirements for the Registration of Pharmaceuticals for Human Use (ICH), representing the pharmaceutical industries and regulatory agencies of the EU, Japan and the USA, agreed that rodent initiation-promotion models could be used. The ICH considered an assay for the detection of hepatocarcinogens that employs an initiator followed by several weeks of exposure to a promoter. Another multi-organ carcinogenesis model involves the use of up to five initiators followed by several months of exposure to the test substance (24).

\section{Pharmacokinetics, pharmacodynamics, and short- term to medium-term rodent and human studies}

With respect to human pharmaceuticals, Ashby (25) proposed that an appreciation of chemical structure and anticipated pharmacokinetics (absorption, distribution, metabolism and excretion) and pharmacodynamics (drug mechanism of action), combined with the short-term evaluation of properties such as genetic, tissue-specific and rodent toxicity, would permit confident predictions of genotoxic and/or non-genotoxic carcinogenic potential, and that the resource-intensive two-year, two-species protocol should, in most cases, be abandoned. Monro (26) similarly proposed the use of in vivo genotoxicity studies combined with three-month to six-month, two-species toxicological studies and phase I and II clinical trials, with the aim of identifying carcinogenic risk factors such as genotoxicity, immunosuppression, hormonal activity or chronic irritation/ inflammation. At least one of these properties was possessed by all of the 19 pharmaceuticals considered to be definite human carcinogens by the IARC. Hence, Monro concluded that the conventional rodent bioassay is redundant.

\section{Non-animal alternatives}

Other investigators have proposed the greater use of non-animal assays in conjunction with, or to replace the conventional rodent bioassay. The non-animal assays currently in existence or under development include quantitative structure-activity relationship (QSAR) systems, in vitro assays, the use of cDNA microarrays to detect changes in gene expression, human clinical trials and epidemiological research. The existing data, much of which remains unavailable within the proprietary files of pharmaceutical and chemical companies, could also be better shared.

\section{SARs and QSARs}

Structure-activity relationships (SARs) predict biological activities such as carcinogenesis, based on the presence of molecular substructures or other chemical moieties which confer biological activity on the parent molecule. Quantitative structureactivity relationships (QSARs) are mathematical descriptions of the relationships between the physicochemical properties of molecules and their biological activities (27).

In 1991, Ashby and Tennant (28) showed that the presence of aromatic amino/nitro-groups, alkylating agents and certain other chemical groups increased the likelihood of rodent carcinogenesis, in a survey of 301 chemicals tested in the NTP. Cronin et al. (29) described a number of electrophilic molecular substructures common to a range of potentially multi-species toxicities, including mutagenicity and carcinogenicity.

Despite initial disappointments, more-recent QSAR databases have been shown to be very useful for predicting the carcinogenicity of test compounds. Matthews and Contrera (30) described the beta-test evaluation of a QSAR computerised system that demonstrated $97 \%$ sensitivity for rodent carcinogens and 98\% specificity for non-carcinogens. When compared to conventional rodent bioassays, computerised SAR and QSAR [(Q)SAR] analyses also have the marked advantages of being very rapid and relatively inexpensive.

\section{In vitro assays}

In vitro assays, involving bacterial, yeast, protozoan, mammalian and human cell cultures, can all contribute information toward a weight-of-evidence characterisation which is sufficient to render the rodent bioassay unnecessary. Brusick (31) found a correlation of approximately $90 \%$ between in vitro microbial mutagenesis and mammalian carcinogenic properties for a large array of chemicals. Tennant et al. (32) successfully predicted the outcomes of $86 \%$ of 44 chemicals undergoing carcinogenicity testing by the NTP, by using the Salmonella mutagenicity and sub-acute (90-day) rodent toxicity tests combined with chemical structural information. The Ames Salmonella typhimurium reverse mutation and chromosomal aberration (CA) genotoxicity assays have been accepted by regulatory agencies for many years (33).

Cell transformation assays detect morphological changes that provide the earliest phenotypically identifiable signs of carcinogenicity. These assays were comprehensively reviewed by Combes et al. (34), and the Syrian hamster embryo (SHE) cell transformation assay has since been described as the most predictive short-term assay for rodent carcinogens (35). It detects morphological cell transformation - the earliest phenotypically identifiable stage in carcinogenesis. Pienta et al. (36) showed a 91\% correlation between the morphological transformation of SHE cells, despite their prior cryo- 
preservation, and the reported carcinogenic activity of numerous carcinogenic and non-carcinogenic chemicals. The particular advantage of the SHE assay in comparison to other in vitro assays, is its ability to detect some non-genotoxic chemicals, as well as genotoxic carcinogens (37-38).

The SHE assay is still undergoing improvement. Most of the difficulties encountered in earlier versions have been overcome by culturing SHE cells at pH $6.7(35,39)$. In a study on 56 chemicals (30 carcinogens, 18 non-carcinogens, 8 inconclusive), LeBoeuf et al. (40) reported an overall concordance of $85 \%(41 / 48)$ between the $\mathrm{pH} 6.7 \mathrm{SHE}$ cell transformation assay and rodent bioassay results, with a sensitivity of $87 \%(26 / 30)$ and a specificity of $83 \%$ (15/18). Furthermore, the assay exhibited a sensitivity of $78 \%$ (14/18) for Salmonella negative carcinogens, demonstrating its ability to detect non-mutagenic carcinogens. Both 24-hour and 7day exposures were used. Mauthe et al. (37) described the testing of the SHE assay during the Health and Environmental Sciences Institute (HESI) branch of the International Life Sciences Institute (ILSI) Alternative Carcinogenicity Testing (ACT) collaboration, which began in 1996. A total of 19 ILSI compounds were tested in the SHE assay - 16 compounds were either known rodent carcinogens and/or human carcinogens, and three were non-carcinogens. The overall concordance between the SHE assay and rodent bioassay results was $89 \%$ (17/19), whereas concordance with known or predicted human carcinogens was a more disappointing 37\% (7/19). However, Zhang et al. (35) demonstrated the potential for further improvement, by showing a 1.4-2.5-fold increase in sensitivity when the cellular incubation time was reduced from the usual 24 hours to less than six hours, prior to seeding onto feeder layers.

Batteries of several in vitro assays offer potential as screening tests. Kirkland et al. (41) examined the ability of a battery of three of the most commonly used in vitro genotoxicity tests, namely, the Ames Salmonella typhimurium, the mouse lymphoma assay (MLA), and in vitro micronucleus (MN) or CA tests, to correctly identify rodent carcinogens, by using a large database of over 700 chemicals compiled from the CPDB, NTP, IARC databases and other publications.

Of 553 rodent carcinogens for which valid genotoxicity data were available, $93 \%$ gave positive results in at least one of the three tests, indicating a high sensitivity of the test battery for rodent carcinogens. Only 9.2\% (19/206) of the rodent carcinogens tested in all three tests gave consistently negative results, and most of these were either nongenotoxic carcinogens (liver enzyme inducers, peroxisome proliferators or hormonal carcinogens), were considered of minimal relevance to humans, or were extremely weak (presumed) genotoxic carcinogens.
The specificity of the Ames test for rodent carcinogens was reasonable (74\%), but both the mammalian cell tests showed very low specificity (below $45 \%$ ), which declined to extremely low levels when they were combined as a battery. When all three tests were performed, $75-95 \%$ of rodent non-carcinogens gave false positive results in at least one test in the battery.

However, by adopting relative predictivity (RP) ratios of true:false results, it was established that positive results in all three tests indicated that the test chemical was more than three times likelier to be a rodent carcinogen than a non-carcinogen, and conversely, that negative results in all three tests indicated more than twice the likelihood of rodent non-carcinogenicity than carcinogenicity. Hence, Kirkland et al. recommended the use of $\mathrm{RP}$ ratios to assess the likelihood of carcinogenicity or non-carcinogenicity for chemicals with batteries of positive or negative results.

The very short time-frames (hours to days), large financial savings, and tiny quantities (micrograms to nanograms) of test chemical required by in vitro assays, all offer strong logistical advantages over the traditional rodent bioassays. However, despite such obvious logistical advantages, the use of in vitro cell cultures is limited by concerns that they do not adequately mimic the response of in vivo cells at the target site within humans. Such concerns can be minimised by using human primary cells, and complex organotypic culture systems, with cofactors and metabolic supplements added to increase longevity and maintain cellular differentiation (18). Nevertheless, continuing problems with human cell transformation systems require further development (34).

The possibilities for in vitro testing will continue to expand with future research. Lichtenberg-Frate et al. (42) demonstrated the genotoxic and cytotoxic sensitivities of a genetically modified yeast (Saccharomyces cerevisiae) assay, which used a yeast-optimised version of green fluorescent protein (GFP) fused to the RAD54 yeast promoter, which is activated upon DNA damage. The result was green fluorescence in the presence of several genotoxic test compounds. Thereafter known as "GreenScreen", this assay permits high throughput testing and requires only minimal quantities of test substances.

The spectrum of compounds detected by GreenScreen is somewhat different to that detected by bacterial genotoxicity assays, hence, as Cahill et al. (43) propose, this assay, in combination with a high throughput bacterial screen and an in silico SAR screen, might provide an effective battery of genotoxicity screening tests for regulatory purposes. An investigation of the ability of GreenScreen to accurately identify carcinogens, and its potential for incorporation into carcinogenicity screening batteries, therefore seems to be warranted. 


\section{cDNA microarrays}

cDNA microarrays, containing hundreds or thousands of microscopic spots of complementary DNA (cDNA) transcripts of mRNA templates (from which the non-coding intron sequences of the original DNA have been excised), hold particular promise for detecting changes in gene expression caused by carcinogens or other toxins (toxicogenomics), long before more invasive effects arise. Unlike previous methods, such as the analysis of mutation frequency by phenotypic selection, analysis of transcription (mRNA) by Northern blotting, or analysis of translation (proteins) by Western blotting, microarrays offer the ability to examine many genes simultaneously, and to characterise phenotypic changes in whole cells or organs.

Although the use of cDNA microarrays for the detection of carcinogens is very recent, early studies have yielded promising results. Lee et al. (44) demonstrated alterations in the gene expression profiles of mouse lymphoma cells when exposed to three genotoxic compounds, namely, hydroxyurea (a carcinogen); p-anisidine (a non-carcinogen); and paclitaxel (carcinogenicity unknown). Seven genes were consistently upregulated and 12 were downregulated more than two-fold by the three genotoxins. By using additional genes, the expression pattern induced by the genotoxic non-carcinogen $p$ anisidine could readily be distinguished from that associated with the genotoxic carcinogen, hydroxyurea, while the profile for paclitaxel was observed to be more similar to that of the genotoxic non-carcinogen.

Particularly exciting, given the current scarcity of suitable alternative models, is the ability of cDNA microarrays to detect non-genotoxic carcinogens (45). After exposing rat hepatocytes to several rodent genotoxic and non-genotoxic carcinogens, as well as to two non-carcinogenic hepatotoxicants, during 5-day, repeat-dose in vivo studies, Kramer et al. (46) hybridised fluorescently-labelled probes generated from liver mRNA against rat $\mathrm{cDNA}$ microarrays. Correlation of the resulting data with the estimated carcinogenic potential of each compound and the dose level, identified several candidate molecular markers of rodent non-genotoxic carcinogenicity, including transforming growth factor-beta-stimulated clone 22 , and $\mathrm{NAD}(\mathrm{P}) \mathrm{H}$ cytochrome P450 oxidoreductase.

However, microarray technology remains in its infancy, and several existing limitations would benefit from further research and development. Clearly, not all genes can be included in microarrays, so the hybridisation results will only represent a subset of the global changes in gene expression. Care must be taken to ensure that the array chosen is appropriate for the hypothesis under investigation, lest important changes in expression be missed. Despite this limitation, the major challenge of microarray work remains the ability to convert a long list of expression results into an interpretable form, necessitating further research into data analysis (47).

\section{Epidemiological research}

Increased epidemiological research, linking cancer incidences with exposure factors in human populations, would identify more likely human carcinogens and presumed non-carcinogens, thereby increasing the data set available for validation studies and for (Q)SAR predictive systems. Currently, despite our extensive tradition of animal testing, too little is known about the human carcinogenicity or non-carcinogenicity of chemicals. Many of the substances classified as definite human carcinogens by the IARC are human viruses, radioisotopes, mixtures, or exposure circumstances not amenable to testing (48). Furthermore, most epidemiological studies for carcinogens are currently performed on substances already known to be human carcinogens (49).

Cancer Centres should be funded, to establish tumour registries aimed at identifying new information on lifestyle, occupational, environmental and medical carcinogens. Post-marketing surveillance of human pharmaceuticals, with mandatory reporting of adverse side effects, is already required in most jurisdictions, and this should be fully utilised.

Increased research should be conducted with the goal of identifying biomarkers of carcinogenicity, such as chromosomal alterations, the presence of mutagens in body fluids, cellular markers of exposure (50), and the presence of carcinogen activating and detoxifying enzymes (51). This would facilitate biomonitoring for early signs of carcinogenicity in human populations.

\section{Data sharing and evaluation}

All existing data about a test substance should be collated and examined in a critical and unbiased fashion, to determine which, if any, remaining tests are scientifically justified, prior to any actual testing. Contrary to the public interest, many existing data remain within pharmaceutical and chemical company files, excluded from the public domain for commercial reasons. The Carcinogenicity and Genotoxicity eXperience (CGX) database, freely offered on the internet by the scientific education charity, LHASA Limited (see http://www.lhasalimited.org/index.php?cat $=4 \&$ sub_cat $=83$ ), provides a large number of published genotoxicity results for rodent carcinogens and non-carcinogens, and is an example of how such information might be organised and freely shared. 


\section{A combination testing protocol}

The conventional rodent bioassay takes upwards of two years to produce results of poor human specificity, and consequently of inadequate predictivity, and is very costly in terms of finances, skilled personnel hours and animal lives. We propose its replacement with the following protocol, based on a tiered combination of alternative assays.

1. Before any assay is conducted, all existing information about the test compound should be collated and reviewed in a critical and unbiased fashion, to determine what, if any, further testing is scientifically justified.

2. Initial screens should include (Q)SAR computerised systems, cell or tissue cultures, and cDNA microarrays, where possible. (Q)SAR systems should be used to identify and estimate the toxic effects of specific chemical groups. The Ames, SHE cell transformation, Saccharomyces GreenScreen, human basal and target organ cell or tissue culture assays, and other appropriate in vitro screening assays, should be fully utilised to seek evidence of cytotoxicity, mutagenicity, genotoxicity and cell transformation. Well-chosen and well-conducted cDNA microarray assays of genotoxicity and non-genotoxicity should be analysed for changes in genetic expression.

3. Following these initial screens, human toxicological studies using barrier models and biological simulations, microdosing and non-invasive biomarker analyses, should be appropriately selected, to model toxicokinetics and to estimate target organ concentrations.

4. In the case of human pharmaceuticals, and nonpharmaceuticals for which a human carcinogenicity assessment is also considered of high importance, and for which human carcinogenicity or other toxicity is not already suggested on the basis of data acquired through the other methods specified in stages $1-3$, limited human trials utilising fully-informed and consenting volunteers (phase I, II and III human clinical trials in the case of pharmaceuticals) might be conducted with considerable caution, commencing with microdoses.

Properly collating and examining the more-targeted data obtained through such a testing scheme for evidence of carcinogenic risk factors such as genotoxicity, immunosuppression, hormonal activity or chronic irritation/inflammation, is likely to yield a weight-of-evidence characterisation of superior human predictivity to that currently offered by the conventional rodent bioassay. Additional advantages include the likelihood of greater insights into mechanisms of carcinogenesis, and substantial saving of financial, human and animal resources.

\section{Further research}

The further development, validation and implementation of some of these alternative assays will no doubt require a redistribution of funding. Areas that clearly merit further research, development and validation include the following:

1. (Q)SAR computerised systems, particularly for initial screening, should be further developed and expanded from their traditional reliance on chemical analogues to include information on the structural properties of cellular receptors which facilitate toxicity, as this information becomes available. Toxicity testing data should be used retrospectively to enlarge the (Q)SAR databases.

2. Cell and tissue assays, particularly those involving human cell lines, the SHE cell transformation assay, other assays sensitive to nongenotoxic carcinogens, and the Saccharomyces GreenScreen assay, should be further developed, validated and implemented. The availability of human cells and tissues for toxicity testing should be increased.

3. Research into improving the reproducibility and interpretation of cDNA microarray data should continue.

4. Predictive biomarkers of toxicity should be identified through genomic, proteomic and clinical research, thereby permitting the speedier generation of results, well prior to the onset of neoplasia and enabling biomonitoring for early signs of carcinogenicity in human populations.

5. Increased human epidemiological research should be conducted, in order to identify more known human carcinogens and presumed noncarcinogens, thereby increasing the dataset available for validation studies and (Q)SAR predictive systems. Cancer Centres should be financially supported to establish tumour registries focused on identifying new human carcinogens, and the post-marketing surveillance of human pharmaceuticals should be fully utilised.

\section{Validation and regulatory acceptance}

Despite the 1997 ICH recommendations, and the criticisms of numerous experts, modernisation of the bioassay protocols has been painfully slow. Battershill and Fielder (52) demonstrated that the 
published genotoxicity data were not sufficient to allow a full assessment of the mutagenic potential of $75 \%(57 / 76)$ of the CPDB chemicals for which bioassay data existed, despite the clear value of such data in interpreting bioassay results, and the far greater resources required for conducting the bioassays themselves.

Although a slowly increasing number of alternative protocols are being submitted to regulatory agencies (53-54), and although the US FDA is accepting genetically-modified mouse data as part of the safety assessment for selected pharmaceuticals (18), for the most part, fear of lack of acceptance of alternatives by regulatory agencies is discouraging the use of alternative assays. Consequently, the conventional two-year, both-genders, two-rodent species (usually, mice and rats) bioassay persists, despite extensive criticism centred on its very poor human specificity, and its subsequent inability to meet the stringent human validation standards required of alternative protocols.

Clearly, regulatory agencies should be required to consider data from promising existing and new alternative testing methodologies, including (Q)SAR computerised systems, appropriate in vitro assays, cDNA microarrays, human toxicological studies and clinical trials, and biological simulations, alongside conventional rodent bioassay data. The validation of promising alternative assays should be accorded a high priority, and once they have been satisfactorily and independently validated, regulators should be required to accept the data they have provided. Where validation has not yet been completed, regulators should nevertheless incorporate appropriate test data into weight-ofevidence assessments. Regulators should be required to make science-based decisions about various test methods, according to the human sensitivity and specificity data of each of them, rather than continuing to rely on the current testing traditions.

Finally, it is of fundamental importance that the harmonisation of testing requirements be achieved among regulatory agencies, as has been achieved in other circumstances under ICH, with significant reductions in the quantity of pharmaceutical testing required (55).

\section{Conclusions}

Conventional carcinogenicity bioassays take around three years to design, conduct and interpret. Unsurprisingly therefore, by 1998 , only about 2000 $(2.7 \%)$ of the 75,000 industrial chemicals then in use and listed in the EPA Toxic Substances Control Act inventory, had been tested for carcinogenicity (56). The cost of testing just these $2.7 \%$ of industrial chemicals in use was hundreds of millions of dollars (23), millions of skilled personnel hours (57), and millions of animal lives (57-58).
Despite this enormous investment of resources, however, the poor human specificity, and hence, the inadequate predictivity, of animal carcinogenicity data, has been illustrated by several investigators (3-10). The reasons for this are numerous. When subjected to careful scrutiny by the IARC, the majority of animal carcinogenicity studies have been found to be scientifically inadequate (56). However, the very high level of scientific rigour required in order that potentially useful data can be derived is only the first barrier that must be crossed. Numerous serious biological obstacles remain, which render it profoundly difficult, if not impossible, to accurately extrapolate human carcinogenic hazards from animal data (59).

Some investigators have proposed modifications to the conventional rodent bioassay, including the elimination of mice, the use of genetically-altered or neonatal mice, decreased time-frames, initiationpromotion models, and the greater incorporation of toxicokinetic and toxicodynamic assessments. Others have proposed the greater use of non-animal assays in conjunction with, or instead of, the conventional rodent bioassay. Non-animal assays currently in existence or in the course of development include (Q)SAR computerised systems, in vitro assays, the use of cDNA microarrays to detect changes in gene expression, human clinical trials, and epidemiological research. Existing data, much of which remains unavailable within pharmaceutical and chemical company files, should also be better shared.

In contrast with animal bioassays, both the human specificity and sensitivity of alternatives such as (Q)SAR computerised systems and in vitro assays are very promising. Non-animal alternatives may also yield results nearly instantaneously, in the case of $(\mathrm{Q}) \mathrm{SAR}$ computerised systems, or in as little as six hours in the case of the enhanced SHE in vitro protocols, compared with two years for conventional rodent bioassays. Other advantages include potentially enormous savings of financial and personnel resources, substantial replacement of animal use, and requirements for only tiny quantities of test chemicals.

Inexplicably, however, in the face of their very substantial potential for increasing human specificity, predictivity and overall efficiency, the regulatory agencies have been frustratingly slow to adopt alternative protocols, preferring to cling to the bioassay traditions that yield results of poor human specificity and predicitivity, at great cost, and after two or more years.

With the 1996 repeal of the Delaney amendment to the US Federal Food, Drug and Cosmetic Act, which had outlawed additives demonstrably carcinogenic in many species, the major US legislative impetus for animal carcinogenicity testing is gone (60). In order to minimise the enormous cost of can- 
cer to society, a substantial redirection of resources away from the very slow and resource-intensive rodent bioassay, into the further development and implementation of non-animal alternative assays, is strongly justified and urgently required.

\section{Acknowledgement}

This research was partly funded by the Physicians Committee for Responsible Medicine, Washington DC, USA.

Received 6.8.05; received in final form 18.12.05; accepted for publication 19.12.05.

\section{References}

1. Huff, J. (1999). Long-term chemical carcinogenesis bioassays predict human cancer hazards. Issues, controversies, and uncertainties. Annals of the New York Academy of Sciences 895, 56-79.

2. Knight, A., Bailey, J. \& Balcombe, J. (2006). Animal carcinogenicity tests: 1 . poor human predictivity. ATLA 34, 19-27.

3. Hogan, K.A. (2000). Characterization of Data Variability and Uncertainty: Health Effects Assessments in the Integrated Risk Information System (IRIS). EPA/635/R-00/005A. 12pp. National Center for Environmental Assessment, Washington DC, USA: Environmental Protection Agency, Office of Research and Development.

4. Tomatis, L. \& Wilbourn, J. (1993). Evaluation of carcinogenic risk to humans: the experience of IARC. In New frontiers in cancer causation. (ed. O.H. Iversen). pp. 371-387. Washington DC, USA: Taylor and Francis.

5. Haseman, K. (2000). Using the NTP database to assess the value of rodent carcinogenicity studies for determining human cancer risk. Drug Metabolism Reviews 32, 169-186.

6. Gold, L.S., Slone, T.H. \& Ames, B.N. (1998). What do animal cancer tests tell us about human cancer risk? Overview of analyses of the carcinogenic potency database. Drug Metabolism Reviews 30, 359-404.

7. Rall, D.P. (2000). Laboratory animal tests and human cancer. Drug Metabolism Reviews 2, 119-128.

8. Ashby, J. \& Purchase, I.F. (1993). Will all chemicals be carcinogenic to rodents when adequately evaluated? Carcinogenesis 8, 489-495.

9. Fung, V., Barrett, J. \& Huff, J. (1995). The carcinogenesis bioassay in perspective: Application in identifying human hazards. Environmental Health Perspectives 103, 680-683.

10. Ennever, F.K. \& Lave, L.B. (2003). Implications of the lack of accuracy of the lifetime rodent bioassay for predicting human carcinogenicity. Regulatory Toxicology and Pharmacology 38, 52-57.

11. Purchase, I.F.H. (1983). Carcinogenicity. In Animals and Alternatives in Toxicity Testing (ed. M. Balls, R.J. Riddell \& A.N. Worden), pp. 109-126. London, UK: Academic Press.

12. Alden, C.L., Smith, P.F., Piper, C.E. \& Brej, L. (1996). A critical appraisal of the value of the mouse cancer bioassay in safety assessment. Toxicologic Pathology 24, 722-725.

13. Van Oosterhout, J.P., Van der Laan, J.W., De Waal, E.J., Olejniczak, K., Hilgenfeld, M., Schmidt, V. \& Bass, R. (1997). The utility of two rodent species in carcinogenic risk assessment of pharmaceuticals in Europe. Regulatory Toxicology and Pharmacology 25, 6-17.

14. Anon. (1983). Report of the FRAME Toxicity Committee. In Animals and Alternatives in Toxicity Testing (ed. M. Balls, R.J. Riddell \& A.N. Worden), pp. 501-540. London, UK: Academic Press.

15. Goodman, J.I. (2001). A perspective on current and future uses of alternative models for carcinogenicity testing. Toxicologic Pathology 29, Suppl. 1, 173-176.

16. Thompson, K.L. \& Sistare, F.D. (2003). Selection of drugs to test the specificity of the Tg.AC assay by screening for induction of the gadd153 promoter in vitro. Toxicological Sciences 74, 260-270.

17. Cannon, R.E. (2003). The Tg.AC mouse model passes test by failing to respond. Toxicological Sciences $\mathbf{7 4}$, 233-234.

18. Combes, R., Schechtman, L., Stokes, W.S. \& Blakey, D. (2002). The international symposium on regulatory testing and animal welfare: recommendations on best scientific practices for subchronic/chronic toxicity and carcinogenicity testing. ILAR Journal 43, Suppl. 1, 112-117.

19. Cohen, S.M. (2001). Alternative models for carcinogenicity testing: weight of evidence evaluations across models. Toxicologic Pathology 29, Suppl. 1, 183-190.

20. McClain, R.M., Keller, D., Casciano, D., Fu, P., MacDonald, J., Popp, J. \& Sagartz, J. (2001). Neonatal mouse model: review of methods and results. Toxicologic Pathology 29, Suppl. 1, 128-137.

21. Grice, H.C. \& Burek, J.D. (1984). Age-associated (geriatric) pathology: its impact on long-term toxicity studies. In Current Issues in Toxicology (ed. H.C. Grice), pp. 57-107. New York, NY, USA: Springer Verlag.

22. Davies, T.S., Lynch, B.S., Monro, A.M., Munro, I.C. \& Nestmann, E.R. (2000). Rodent carcinogenicity tests need be no longer than 18 months: an analysis based on 210 chemicals in the IARC Monographs. Food and Chemical Toxicology 38, 219-235.

23. Conolly, R.B., Reitz, R.H., Clewell, H.J. 3rd \& Andersen, M.E. (1988). Pharmacokinetics, biochemical mechanism and mutation accumulation: a comprehensive model of chemical carcinogenesis. Toxicology Letters 43, 189-200.

24. International Federation of Pharmaceutical Manufacturers Association (1997). ICH Harmonised Tripartite Guideline: Testing for Carcinogenicity of Pharmaceuticals. 6pp. Geneva, Switzerland: IFPMA. Website http://www.ich.org (Accessed 01.03.04).

25. Ashby, J. (1996). Alternatives to the 2-species bioassay for the identification of potential human carcinogens. Human and Experimental Toxicology 15, 183-202.

26. Monro, A. (1996). Are lifespan rodent carcinogenicity studies defensible for pharmaceutical agents? Experimental and Toxicologic Pathology 48, 155-166.

27. Comber, M.H., Walker, J.D., Watts, C. \& Hermens, J. (2003). Quantitative structure-activity relationships for predicting potential ecological hazard of organic chemicals for use in regulatory risk assessments. Environmental Toxicology and Chemistry $\mathbf{2 2}$, 
$1822-1828$.

28. Ashby, J. \& Tennant, R.W. (1991). Definitive relationships among chemical structure, carcinogenicity and mutagenicity for 301 chemicals tested by the U.S. NTP. Mutation Research 257, 229-306. Erratum in Mutation Research 1994 317, 175.

29. Cronin, M.T., Dearden, J.C., Walker, J.D. \& Worth, A.P. (2003). Quantitative structure-activity relationships for human health effects: commonalities with other endpoints. Environmental Toxicology and Chemistry 22, 1829-1843.

30. Matthews, E.J. \& Contrera, J.F. (1998). A new highly specific method for predicting the carcinogenic potential of pharmaceuticals in rodents using enhanced MCASE QSAR-ES software. Regulatory Toxicology and Pharmacology 28, 242-264.

31. Brusick, D.J. (1977). In vitro mutagenesis assays as predictors of chemical carcinogenesis in mammals. Clinical Toxicology 10, 79-109.

32. Tennant, R.W., Spalding, J., Stasiewicz, S. \& Ashby, J. (1990). Prediction of the outcome of rodent carcinogenicity bioassays currently being conducted on 44 chemicals by the National Toxicology Program. Mutagenesis 5, 3-14.

33. Dearfield, K.L., Auletta, A.E., Cimino, M.C. \& Moore, M.M. (1991). Considerations in the U.S. Environmental Protection Agency's testing approach for mutagenicity. Mutation Research 258, 259-283.

34. Combes, R., Balls, M., Curren, R., Fischbach, M., Fusenig, N., Kirkland, D., Lasne, C., Landolph, J., LeBoeuf, R., Marquardt, H., McCormic, J., Muller, L., Rivedal, E., Sabbioni, E., Tanaka, N., Vasseur, P. \& Yamasaki, H. (1999). Cell transformation assays as predictors of human carcinogenicity: The report and recommendations of ECVAM Workshop 39. ATLA 27, 745-767.

35. Zhang, H., Borman, H.D. \& Myhr, B.C. (2004). Enhancement of the morphological transformation of Syrian hamster embryo (SHE) cells by reducing incubation time of the target cells. Mutation Research 548, 1-7.

36. Pienta, R.J., Poiley, J.A. \& Lebherz, W.B. 3rd (1977). Morphological transformation of early passage golden Syrian hamster embryo cells derived from cryopreserved primary cultures as a reliable in vitro bioassay for identifying diverse carcinogens. International Journal of Cancer 19, 642-655.

37. Mauthe, R.J., Gibson, D.P., Bunch, R.T. \& Custer, L. (2001). The Syrian hamster embryo (SHE) cell transformation assay: review of the methods and results. Toxicologic Pathology 29, Suppl. 1, 138-146.

38. Amacher, D.E. \& Zelljadt, I. (1983). The morphological transformation of Syrian hamster embryo cells by chemicals reportedly nonmutagenic to Salmonella typhimurium. Carcinogenesis 4, 291-295.

39. Kerckaert, G.A., LeBoeuf, R.A. \& Isfort, R.J. (1998) Assessing the predictiveness of the Syrian hamster embryo cell transformation assay for determining the rodent carcinogenic potential of single ring aromatic/nitroaromatic amine compounds. Toxicological Sciences 41, 189-197. Erratum in Toxicological Sciences 1998 46, 420.

40. LeBoeuf, R.A., Kerckaert, G.A., Aardema, M.J., Gibson, D.P., Brauninger, R. \& Isfort, R.J. (1996). The $\mathrm{pH}$ 6.7 Syrian hamster embryo cell transformation assay for assessing the carcinogenic potential of chemicals. Mutation Research 356, 85-127.

41. Kirkland, D., Aardema, M., Henderson, L. \& Muller, L. (2005). Evaluation of the ability of a battery of three in vitro genotoxicity tests to discriminate rodent carcinogens and non-carcinogens I. Sensitivity, specificity and relative predictivity. Mutation Research 584, 1-256.

42. Lichtenberg-Frate, H., Schmitt, M., Gellert, G. \& Ludwig, J. (2003). A yeast-based method for the detection of cyto- and genotoxicity. Toxicology in Vitro 17, 709-716.

43. Cahill, P.A., Knight, A.W., Billinton, N., Barker, M.G., Walsh, L., Keenan, P.O., Williams, C.V., Tweats, D.J. \& Walmsley, R.M. (2004). The GreenScreen genotoxicity assay: a screening validation programme. Mutagenesis 19, 105-119.

44. Lee, M., Kwon, J., Kim, S.N., Kim, J.E., Koh, W.S., Kim, E.J., Chung, M.K., Han, S.S. \& Song, C.W. (2003). cDNA microarray gene expression profiling of hydroxyurea, paclitaxel, and p-anisidine, genotoxic compounds with differing tumorigenicity results. Environmental and Molecular Mutagenesis 42, 91-97.

45. Goodman, J.I. (2001). A perspective on current and future uses of alternative models for carcinogenicity testing. Toxicologic Pathology 29, Suppl. 1, 173-176.

46. Kramer, J.A., Curtiss, S.W., Kolaja, K.L., Alden, C.L., Blomme, E.A., Curtiss, W.C., Davila, J.C., Jackson, C.J. \& Bunch, R.T. (2004). Acute molecular markers of rodent hepatic carcinogenesis identified by transcription profiling. Chemical Research in Toxicology 17, 463-470.

47. Docterman, K.E. \& Smith, S.M. (2002). Of meis and men: lessons from a microarray study of teratogen action. Teratology 66, 217-223.

48. Ennever, F.K. \& Lave, L.B. (2003). Implications of the lack of accuracy of the lifetime rodent bioassay for predicting human carcinogenicity. Regulatory Toxicology and Pharmacology 38, 52-57.

49. Karstadt, M. (1998). Availability of epidemiologic data for chemicals known to cause cancer in animals: an update. American Journal of Industrial Medicine 34, 519-525.

50. Cone, J.E. \& Rosenberg, J. (1990). Medical surveillance and biomonitoring for occupational cancer endpoints. Occupational Medicine 5, 563-581.

51. Besaratinia, A., Kleinjans, J.C. \& Van Schooten, F.J. (2002). Biomonitoring of tobacco smoke carcinogenicity by dosimetry of DNA adducts and genotyping and phenotyping of biotransformational enzymes: a review on polycyclic aromatic hydrocarbons. Biomarkers 7, 209-229.

52. Battershill, J.M. \& Fielder, R.J. (1998). Mouse-specific carcinogens: an assessment of hazard and significance for validation of short-term carcinogenicity bioassays in transgenic mice. Human and Experimental Toxicology 17, 193-205.

53. International Life Sciences Institute, Health and Environmental Sciences Institute (2003). ILSI HESI Alternatives to Carcinogenicity Testing Committee Workshop on the Utility of Transgenic Assays for Risk Assessment. Washington DC, USA: ILSI, HESI. Website http://hesi.ilsi.org/index.cfm? pubentityid $=48$ (Accessed 01.03.04).

54. MacDonald, J., French, J.E., Gerson, R.J., Goodman, J., Inoue, T., Jacobs, A., Kasper, P., Keller, D., Lavin, A., Long, G., McCullough, B., Sistare, F.D., Storer, R. \& Van Der Laan, J.W. (2004). The utility of genetically modified mouse assays for identifying human carcinogens: a basic understanding and path forward. Toxicological Sciences 77, 188-194.

55. Osterberg, R.E. (2001). ICH approaches to reducing 
animal use. In TestSmart-Pharmaceuticals: An Efficient and Humane Approach to Predictors of Potential Toxic effects of Drugs, held in Baltimore, Maryland, May 7-9, 2001. Baltimore, Maryland, USA: The Center for Alternatives to Animal Testing. Website http://caat.jhsph.edu/programs/workshops/ testsmart/pharm/osterberg.htm (Accessed 13.01.05).

56. Epstein, S.S. (1998). The Politics of Cancer Revisited. 770pp. Fremont Center, NY, USA: East Ridge Press.

57. Gold, L.S., Manley, N.B., Slone, T.H. \& Rohrbach, L. (1999). Supplement to the Carcinogenic Potency Database (CPDB): results of animal bioassays published in the general literature in 1993 to 1994 and by the National Toxicology Program in 1995 to 1996. Environmental Health Perspectives 107, Suppl. 4, 527-600.

58. Monro, A.M. \& MacDonald, J.S. (1998). Evaluation of the carcinogenic potential of pharmaceuticals. Opportunities arising from the International Conference on Harmonisation. Drug Safety 18, 309-319.

59. Knight, A., Bailey, J. \& Balcombe, J. (2006). Animal carcinogenicity tests: 2 . obstacles to extrapolation of data to humans. ATLA 34, 29-38.

60. Greek, C.R. \& Greek, J.S. (2000). Sacred Cows and Golden Geese: The Human Costs of Experiments on Animals. 242pp. New York, USA: Continuum International Publishing Group. 